\title{
Diversity and Distributed Agency in the Design and Use of Medical Video-Communication Technologies
}

\author{
Nelly Oudshoorn, Margo Brouns, and Ellen van Oost
}

\section{Introduction}

Imagine a scene in a hospital. In the intensive care unit, a nurse is taking care of a tiny, premature baby that moves restlessly in the incubator. When the nurse has reassured herself that everything is okay, she installs the camera that watches over the baby in the incubator. The signals from the camera are sent to the central control unit in the hospital and to yet another location, thirty miles from the hospital where, connected by means of telephone cables, the parents of the child try to operate the video-communication system they have received from the hospital. If they can manage, they will be able to see their baby without leaving their living room. A fourth location, not materially connected to the three previously mentioned locations but nevertheless important to this story, is the hospital management room. The manager in charge of the introduction of the Baby Watch, as this video apparatus is called, discusses the risks of legal claims against the hospital in the case of when something appears to go wrong while the parents are recording their child's medical treatment via the Baby Watch. What all of these situations have in common is that they represent different contexts of use and users that are part of a heterogeneous network of actors - people as well as objects - that jointly constitute a new practice in the supervision of premature babies.

In this chapter, we aim to analyse the role of the diverse actors - both human and nonhuman - in the development of this new practice, this hybrid, heterogeneous network. In our analysis, the design and use of a new technology are intimately entwined. The notion of script is relevant to conceptualising the connection of design and use. Akrich (1992) introduced this concept to visualise the way in which innovators' representations of users shape technological development. In the design phase, actors construct many different images of users and objectify these representations in technological choices. The very act of identifying specific individuals or groups as users may facili- 
tate or constrain the actual role that specific groups of users are allowed to play in shaping the development and use of technologies. The inscription of representations of users and use in artifacts results in technologies that contain a script: they attribute and delegate specific competencies, actions, and responsibilities to users and technological artifacts. As such, technological artifacts - nonhuman actors - are not neutral actors in the socio-technical configuration, or "hybrid collective" to use Latour's terminology. Like humans, nonhumans can act, have intentions (mediated), can delegate, distribute responsibilities, etc. This symmetrical analysis of humans and nonhumans is one of the most important merits of actor-network theory and has undoubtedly shed new light on the way technology configures practices (medical and other) (Pasveer and Akrich 1996; Lehoux et al. 1999).

Although the script approach, as developed by Akrich, is potentially adequate for our analysis, it tends to neglect the diversity of user groups involved as well as the heterogeneity within these groups. Whereas Akrich briefly mentions that there is usually a variety of different user representations at work in the design process, her semiotic approach does not provide any theoretical reflections or conceptual tools to acknowledge the heterogeneity within user groups and differences in agency between user spokespersons, nor does it specify explicitly how change might be accomplished.

This chapter seeks to explore how technologies may contribute to (a)symmetry in agency and control among users and user spokespersons, i.e., end users, intermediary users, and commissioners. A second and equally important aim is to understand the processes underlying the design of technologies for multiple users.

To do so, we adopt the concept of distributed agency. As actor-network theory suggests, agency is not an a priori given feature of an actor but is the outcome of interactions between the heterogeneous actors in the network. The notion of "distributed agency" emphasises that agency is not located in an individual actor, but is distributed among diverse (human as well as nonhuman) actors that jointly form a collective actor (Gomart and Hennion 1999). Technological artifacts not only distribute agency among people and things, they may also delegate agency to specific groups of users and not to others. We do not set ourselves the task of elaborating a theoretical conceptualisation of this type of agency that does not privilege humans above nonhumans, as classical sociology does. Latour has tried to redefine agency/action to include nonhumans in sociological theory (Latour 1996 2 237) but failed, even in his own estimation (Latour $1996^{\mathrm{b}}, 269$ ). ${ }^{1}$ Instead, we shall elaborate the concept of distributed agency empirically and, based on this exercise, dis- 
cuss the benefits and problems of this concept. We consider distributed agency as a potentially interesting concept because it retains the valuable contribution of actor-network theory, that is, the symmetrical inclusion of nonhumans and humans in sociological analysis, whereas two points of criticism of actor-network theory are obviated: that actor-network theory ignores differences among actors and that it pays exclusive attention to experts, engineers, and producers (Clarke 1998, 267; Star 1991).

Our concern in writing this chapter is to understand how new technologies contribute to the equal/unequal distribution of agency over the diverse user groups. Social issues like equality and inequality and the implicit or explicit exclusion of vulnerable social groups in relation to new technologies are at the core of our research motivation. Our research builds on a feminist tradition in technology studies where attention to users is important. The feminist concern has been, and remains, the virtually exclusive attention paid to experts in actor-network theory ("the executive approach") which tends to preclude knowledge from the vantage point of non-standard positions, including women's voices. Feminist scholars argue that it is important to include the "silent" and "silenced" voices of less-powerful actors than experts and producers in technology studies (Star 1991; Clarke and Montini 1993, Clarke 1998; Saetnan et al. 2000).

This argument reflects a political and theoretical perspective. Politically, feminists argue for an engagement with those individuals and groups who are affected downstream by products of technological innovation (Casper and Clarke 1998). In a situation of multiple user groups, which is often the case in medical technologies, the end-users run the risk of being marginalised in the design process (Berg et al. 1999). A more detailed understanding of the role of users as actors, how they are represented, and how they are endowed with agency in the process of development and use of new technology may provide useful information for the empowerment of users or user spokespersons, such as patient groups and consumer groups, to envisage technologies that match their interests and increase their agency. Theoretically, feminists denounce the idea that science and technology in action begin or end with the actions of scientists and engineers. They emphasise the importance of acknowledging the heterogeneity of actors in networks of technological development and use (Rapp 1998, 48). The concept of distributed agency may well contribute to the development of this theoretical and political perspective.

In this chapter, we shall present our study of the design process of a videocommunication system that enables parents of premature babies to visit, in a 
"virtual" sense, the neonatology ward of a hospital to watch their baby. ${ }^{2}$ The design trajectory encompassed different artifacts. Each artifact was actually put into use, which enables us to describe and analyse the developmental process as an intimate entwining of design and use. In analysing the transformations in the heterogeneous network of actors involved in the design and use of the video-communication system, we first describe the ways in which the script of the technology organises and structures the network in terms of the distribution of control and agency among the different actors, human as well as nonhuman, with a particular interest in asymmetries between the different human actors. We continue by analysing the design processes underlying the development of this technology. What influence do the different user groups have on actual design choices? We conclude by discussing the extent to which the concept of distributed agency is the appropriate tool to account for equalities and inequalities among the heterogeneous actors in shaping technological development.

\section{The birth and development of Baby Watch}

New technological products can have different origins. Whereas some products have been designed in reaction to specific demands or for the purpose of solving specific problems (technology pull), other products are developed because designers want to experiment with different technological possibilities to create new markets (technology push). Baby Watch is typically a product of the latter. For a long time, the Royal Dutch Telephone Company was a public company, and the only provider of telephone services in the Netherlands. This monopoly position was seriously challenged when the Dutch Government decided to give up its ownership and to create conditions for a competitive telecommunications market. As a privatised organisation, Royal Dutch Telephone had to re-orient itself to new clients and future markets. In this re-orientation, they considered video-communication as one of the promising markets. In the early 1990s, the R\&D department of Royal Dutch Telephone developed an algorithm for compressing video images so that they could be transmitted via analogue lines. The firm developed a demonstration set using a laptop computer, and tried to generate experiments and orders for developing new products based on this technology.

The account manager of Royal Dutch Telephone, who served as the permanent intermediary between Royal Dutch Telephone and the Radboud Hospital in Nijmegen and was responsible for creating opportunities to introduce new communications products, presented the demonstration set 
during one of his regular visits to the hospital. Although the performance of the demonstration set was rather limited - only pictures the size of a postage stamp could be transmitted - the hospital's response was positive: "We were looking for this kind of communication". ${ }^{3}$ The Radboud Hospital was interested in developing new services for its clients, a project allowing the hospital to present itself as a modern hospital using the latest technologies with the aim of increasing customer satisfaction. The neonatology ward promised to provide a suitable location to initiate the new services. In the neonatology ward, the medical and caring systems are primarily oriented towards newborn babies, vulnerable due to premature or early birth. The parents worry about the future of their child, the mothers are often weakened by childbirth. The babies end up staying in the academic hospital for quite a long time. The patients come from different places, not only from the immediate neighbourhood of Nijmegen, but even from Germany. The management of the hospital saw a new video-communication system, which enabled parents to have contact with their child from their home, as an adequate tool to create a new communication line between parents and their baby, and as a new service that could attract patients from wider regions.

To Royal Dutch Telephone, the academic hospital was a promising experimental site for developing video-communication systems. Hospitals function in an extensive web of internal and external communication between different groups: medical staff, nurses, patients and their families, students, insurance companies, and governments. This makes the hospital a potential major customer for telecommunication services. The development of a video-communication system thus served the interests of both parties. In early 1994, the Radboud Hospital management and Royal Dutch Telephone, financially supported by two insurance companies, decided to develop a video-communication system for the neonatology ward, called Baby Watch.

The development of this new medical video-communication system resembles the career of Michael Prince: the artifact has lived through quite drastic changes, even to the extent that each product was given a different name. The first system envisaged, Baby Watch, was based on the same technology as the demonstration set, using a laptop, modem and telephone. Not only did the compression software need to be improved in order to produce images of sufficient quality, but a user interface aimed at the specific user group also had to be developed. At the end of 1995, the technical designers could present the first prototype of the Baby Watch system. During 1996 and 1997, this system was implemented and adapted to the specific circumstances at the hospital. In 1998, Baby Watch was replaced by a completely new system, viatv Phone. This system, also developed by Royal Dutch Telephone, used the Tv to show 
the images. It was, however, yet another application of this technology that became the proclaimed commercial video-communication product, "Magic TV", designed to be used in the children's oncology ward. In commercial terms, Magic Tv is Royal Dutch Telephone's most successful product, since it is easier for hospitals to raise funds for young cancer patients.

Altogether, Royal Dutch Telephone developed two different technologies (respectively, a computer-based technology and a Tv-based technology) and three different products: Baby Watch, the viatv Phone, and Magic Tv. The following section analyses the scripts of these three different products.

\section{Delegation and distribution of agency and control by the video- communication systems}

The initial Baby Watch system consisted of a camera attached to the incubator and connected to a server in a room nearby. The parents had a laptop with a specially developed Baby Watch interface and a modem to connect the laptop to the telephone line at home. The telephone line was used for communication between the parents and the nurses and for sending the pictures from the camera to the laptop. The nurses controlled the pictures on a monitor before opening the line. The design is as follows:

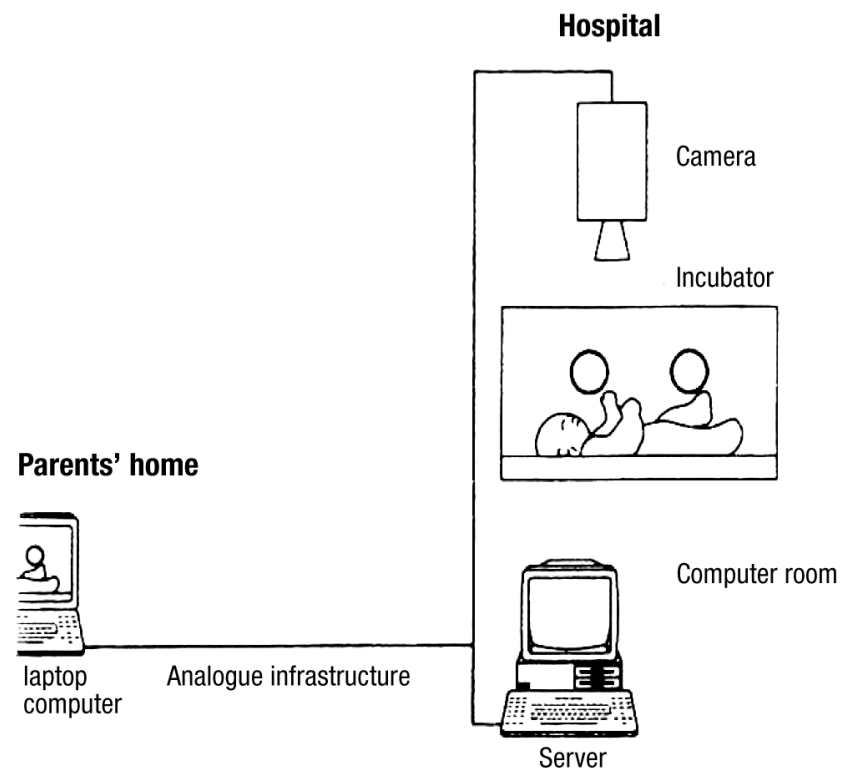


Once this first prototype was set up, most of the responsibilities and control were delegated to the nurses. The parents had to telephone the nurses and ask them to activate the camera. As a result, the agency of the parents was restricted. First of all, they were dependent on the nurses to make the connection. Then the nurse initiated the system and controlled the monitor. If the parents switched on the PC at home, they were then able to watch their child on a laptop screen. A second restriction to the parents' agency was that they never obtained a connection when the baby was being medically treated. Last, but not least, they could only receive images and no sounds. The Baby Watch thus incorporated a clear script that delegated responsibilities, agency, and control to specific users and created dependencies between different groups of users.

After some weeks, it turned out that parents did not use Baby Watch as much as expected. The script of the Baby Watch system was adjusted to remove one of the most important restrictions, the parents' dependence on the nurses to start up the system. In the second prototype of the Baby Watch, the camera was installed permanently at the baby's incubator, and the parents did not need to phone the nurses in order to use the Baby Watch. From that moment on, the parents could watch their baby whenever they wanted, without the intervention of the nurses. However, now control of the images was not delegated only to the nurses, but also to a lid that could cover the camera while the baby was being treated. The responsibility for the lid was fully delegated to the nurses. If, for instance, they forgot to remove the lid after treatment, the parents could potentially become anxious as they tried to see their baby. After these changes, however, the popularity of Baby Watch among the parents clearly increased.

Compared with the first product, the second product, viatv Phone, had two distinctly new features: first, the computers (laptop as well as the server) were replaced by a $\mathrm{TV}$ and a little box that connected the $\mathrm{TV}$ to the phone; and second, the system allowed parents more control of the images. The script of this second product is very similar to the second prototype of the Baby Watch: responsibilities and control are still largely delegated to nurses. When parents started up the viATv system, the nurses had to react to the signal and commence communication by lifting the telephone. At that moment, they also checked the position of the baby and the quality of the images. The nurses' responsibility for covering the camera with a lid during treatment and uncovering it after treatment did not change. However, the script of the viatv Phone shows less-restricted agency for the parents with respect to controlling the images. They could choose between moving or fixed, clear pictures, they could operate a zoom lens, and move the camera (to a limited extent). Just like 
Baby Watch, the viatv Phone system does not broadcast sound. Although the viATv technology does allow the functionality of transmitting sound, this option was ruled out here by disconnecting the sound wires in the camera. Thus, the control of sound from the hospital to the parents' home was, in fact, fully localised within the disconnected wires. Since the viaTv system is interactive, it also enables the hospital to receive images from the parents' home. The responsibility for controlling these images was not realised within the technology but was delegated to the parents. Parents were advised to point the camera at the wall to prevent pictures being sent from their living room to the hospital. Thus, there is a clear asymmetry in the way the viatv Phone script delegates the control over the hospital's privacy and the parents' privacy.

The viatv Phone is the final stage in the development of the video-communication system in neonatology. However, this video-communication system also turned out to be an interesting technology for other user locations. Royal Dutch Telephone decided to develop a new product based on the same technology as viatv, the so-called "Magic Tv", to be used in the children's oncology ward. Although we shall primarily focus our analysis on the products designed for the neonatology ward, Magic TV is relevant to our analysis as it allows us to visualise the specific scripts that were built into the VIATv Phone system used in the neonatology ward. In contrast to the way the VIATv Phone system is used in the neonatology ward, Magic TV offers the possibility for symmetrical communication between the two locations. It is a fully interactive system transmitting images as well as sound, allowing both sides to control the connection and the images. With Magic TV, the cancer patients can continue to interact with their friends, family and school during hospital treatment. Compared with the previous two products, the Magic TV script delegates much less control to the nurses and, again, more autonomy to the parents and, most importantly, to the children.

As we have seen, the various video-communication systems contain quite different scripts in terms of distributing agency, control, and responsibility among people and things, and among the different user groups involved. To understand how these scripts were generated, we shall focus on the design process.

\section{Designing scripts: The co-production of an artifact and its users}

We shall analyse the developmental process of the video-communication system from two perspectives. First, we look at the way in which the different ac- 
tors involved in the design process represented the envisaged users. After all, it is these notions of the user that are objectified into design choices and thus constitute the script of the artifact. But who is the user? In fact, the designers and other actors involved in the development of this new artifact actually considered four different groups of users: the parents, the nurses, the management of the hospital, and the baby. Which representations were made of these user groups? Which techniques were used to represent the users? Second, we look at the way the diverse user groups were able and were allowed to influence the design process. This is really a question of those directly involved by the designers in the design process, but we can go one step further. Since the developmental process of the video-communication system was characterised by a succession of different prototypes that were actually put into use, we are able to question how the distribution of agency, control, and responsibilities in the socio-technical configuration around one prototype may have influenced the design and the script of the next one. The iterative succession of design and use allows us to trace differences between the user representations and the actual user, and to analyse whether or not these differences or mismatches shaped the future design.

A first glance at the design process reveals that there are important differences in the ways in which each group of users was represented in the design process. Whereas nurses and the hospital management were directly involved in decisions shaping the design, because representatives of these groups were part of the committee that was installed to guide the design process, the parents and the babies were represented by nurses and the hospital management, who were entitled to act as spokespersons for these users. Thus, the parents (and babies) were not granted agency to contribute to the negotiations on the design, because the hospital manager and the senior nurse assumed that they would not want to worry about anything else other than their babies. They did not consider an alternative representation of the parents, and decided that the nurses were sufficiently informed on the positions of the parents to represent their preferences and interests. The account manager responsible for the interaction between the hospital and Royal Dutch Telephone did not make an issue of the absence of parents on the committee. As a result, the project committee consisted of three nurses, the head of the neonatology ward (a senior nurse), a doctor, and a representative of Royal Dutch Telephone called "the human-factor specialist", who acted as the project co-ordinator and served as the intermediary between the hospital and both the technical designers and software engineers. 


\section{Parents}

The fact that parents were not considered as relevant actors for participation on the project committee implied that the designers had to rely on other sources to assess the needs and preferences of the future end-users. The design process of the first prototype shows that parents were not consulted at all. The engineers only applied informal techniques to represent the user. ${ }^{4}$ In contrast to what one might expect, informal techniques are widely used in many Information and Communication Technology companies (Oudshoorn 1999). In the case of the Baby Watch project, the major representation technique consisted of inviting others to speak for the users. In this case, nurses were considered adequate spokespersons for the parents. Since nurses had frequent contact with parents on the neonatology ward, they were expected to be able to assess the needs of the end-users. The nurses, too, regarded themselves as adequate spokespersons for the parents: "We thought we knew what they wanted".

Another representation technique frequently used in the design of the first prototype was the so-called I-methodology: engineers used their own ideas and experiences as a first user of computers to generate a representation of the future user. The dominance of these informal representation techniques had a major impact on the script of the first prototype. The user images constructed by the nurses as well as engineers show a very specific representation of the end-users, including a specific assessment of the skills they were expected to have to operate the new artifact. Nurses and engineers represented the parents as computer illiterates: parents were expected to have no experience with computers or IC T products at all. This was precisely the reason why Royal Dutch Telephone considered the development of this new artifact as relevant enough to include it in their $\mathrm{R} \& \mathrm{D}$ agenda. The challenge the designers expected to face was "to develop a computer that did not look like a computer". ${ }^{6}$ At that time, only 50 per cent of the Dutch population were familiar with computers, and Royal Dutch Telephone expected that not all potential end-users of Baby Watch would be capable of handling a PC and the corresponding software program. Baby Watch thus provided a convenient experimental site to explore the conditions and technical features of a computer system that had to be made as user-friendly as possible. The image of users as computer illiterates functioned as an important element in shaping the technological decisions and the script of the system. Based on this user image, the Royal Dutch Telephone decided to design a product that could be operated with a simple click. A second feature of this first prototype shows that designers also actively configured a non-user. Both Royal Dutch Telephone and the 
hospital management did not want any interference from outside, so the designers developed a system that was protected against the more sophisticated user. The result was a fast-running system that could be operated with an on/off switch, but without any possibilities for further action by the users. ${ }^{7}$

Although designers thus put a lot of effort into making a computer that they considered capable of matching the needs and skills of the end-users, the reactions of the parents to the first prototype show a clear mismatch between the presumed attitudes of users towards computers, as represented by the nurses and the engineers, and the actual attitudes of the real users. Whereas engineers and nurses did not consider the computer itself as a possible stumbling block for the end-users (making the computer user-friendly was considered sufficient to solve the expected problem of lack of computer experience), parents turned out to be very reluctant to use the computer at all. During the first weeks of the introduction of Baby Watch, only a handful of parents could be convinced to take the laptop home. In this experimental stage, every week it was difficult to find a parent who wanted to use Baby Watch. Whereas the Telephone Company and hospital management presumed that the Baby Watch would stimulate the quality of the child-parent relationship, many parents were not easily convinced of the usefulness of this kind of communication. Moreover, they saw it as a redefinition of the parental relationship by introducing a technical artifact into the relationship with their vulnerable child. Despite the fact that engineers had put a lot of effort into making the computer as user-friendly as possible, it was, nevertheless, still a computer.

A few weeks after the introduction, the parents were given a voice for the first time via a small questionnaire that was developed by the project co-ordinator. This questionnaire made it very clear why parents were reluctant to use Baby Watch: they feared that the system might create too-remote a relationship between themselves and the baby. Although the questionnaire did not provide information about the ethnicity of the parents involved, the nurses did experience an ethnic disparity. Parents from a non-Western background were more reluctant to use Baby Watch. The nurses felt that, within these families, Baby Watch was considered as an unwelcome, technical intruder into their homes. ${ }^{8}$ To them, some spheres of life should not be "technologised" - especially the area of love and vulnerability. To make Baby Watch acceptable to these parents, the designers had to mask the technological image of the artifact. The fact that the $\mathrm{TV}$ was acceptable to a wider group of the parents shows how this device is no longer considered as technology. Over the last few decades, the $\mathrm{TV}$ has witnessed such a rapid dissemination (most Dutch families, including immigrants, have a TV in their living room) that the artifact has lost its technological image. This is obviously not the case with 
the computer. Differences in attitudes between different cultural groups towards technology had not been anticipated by the engineers.

The questionnaire also informed the designers and the nurses about the barriers parents experienced using Baby Watch. The parents experienced the access to the system as a major barrier to using Baby Watch. The script of the first prototype delegated access responsibilities to the nurses who functioned as gatekeepers. Parents had to phone the nurses in order to operate the system, and despite the fact that parents and nurses had a tradition of making phone calls concerning the well-being of the child, this seemed to be an extra obstacle. Many parents, especially mothers, wanted to watch their child late in the evening and during the night, not quite the time to disturb nurses with a telephone call. Or, as the project co-ordinator concluded: "To ask permission for a video connection unconsciously seems to put up a barrier for the parents". In this respect, there were major differences between the preferences of users as imagined by the designers and the preferences of the actual users. As a result, the system was adapted to the parents' behaviour. Whereas the first prototype operated only on stand-by, the second prototype of Baby Watch worked on a permanent basis and thus increased the parents' agency and control. This was the first time that users, i.e., the parents, had an impact on the actual design of the new technology.

Another mismatch between the users as represented by the designers and the actual users concerned the design choices about the ways in which images should be presented on the screen. At that time, the system was not sophisticated enough to produce clear running pictures, so the designer had to choose between two kinds of application: a clear picture with little movement, or a moving, but more vague image. Relying on the I-methodology, the designer chose a clear picture. Both the engineers and the account manager preferred to have a good view, and they assumed that parents would have the same preference because they would like to recognise their baby. Or as the account manager stated: "We expect the users to demand the same quality as we do". 9 The parents, however, wanted to see their children move, more or less as a sign of health. In the second prototype, the designers therefore adapted the design of the screen to enable parents to select whether they wanted a clear picture or a moving picture. This was the second time that parents exercised an influence on the design.

The design process of the first prototype thus exemplifies the risks that are involved in relying on informal and indirect methods of assessing the needs and skills of users. In a constellation in which all the information is mediated by other agents, spokespersons, and representations, designers run the risk of 
losing crucial information. In this case, this covered information on user preferences for a specific type of artifact (i.e., $\mathrm{TV}$ rather than a computer), information on the user needs in a medical context, and the social preconditions for using the artifact (the relationship between nurses and parents). In this respect, the design of Baby Watch was an exceptional experience for the Royal Dutch Telephone engineers. Usually, IC T products are designed for professional users or for fun - think of the many digital ICT games that have been developed over the last decade. By developing Baby Watch, engineers entered a completely different world where the people using IC т products are experiencing sorrow and grief rather than pleasure or work. Engineers could not anticipate these differences because they had no contact with the parents and did not visit the neonatology ward during the development of the first prototype. It was only during the development of the second prototype that one of the engineers visited the hospital, and this is how he described his experiences:

Suddenly, you realise that this is a completely different world. Here are people with sorrow and grief, they are not sitting and waiting for a new toy. For them, all that matters is the child. This was a dramatic experience for me. We could have known this, but we did not think about it. We are primarily engaged with the technology. ${ }^{10}$

Reflecting on his experiences with the design of this new artifact, the account manager came to a similar conclusion. Due to technical problems with Baby Watch, the account manager visited the parents at home, which provided new information on how to solve technical problems with the PC and the transmitter. Visits to the location of use and consulting the parents via questionnaires were thus only introduced after the development of the first prototype.

\section{Babies}

Just like the parents, the babies were also represented in the design process by spokespersons. There is, however, an important difference between parents and babies. Whereas parents are able to take part in the design process without mediation by spokespersons, babies cannot speak for themselves. They are dependent on spokespersons to communicate with designers and represent them in the design process. In the design of Baby Watch, nurses and hospital management acted as spokespersons for the babies. Nurses represented the baby by articulating their worries about possible negative consequences of the introduction of Baby Watch in the neonatology ward. The nurses con- 
sidered regular visits from the parents to be important to the well-being of the child. They feared that the introduction of Baby Watch would reduce the visits of parents to the hospital because they could simply stay at home and observe their child. The hospital management also acted as spokesperson for the baby, but constructed the opposite image: babies in incubators would benefit from Baby Watch because the device would facilitate the bonding between parents and babies. During the first months after the introduction of Baby Watch, this representation of the baby as someone who benefits from Baby Watch became the dominant image, which was accepted by nurses as well. Most importantly, the frequency of the actual visits did not decrease after the introduction of Baby Watch. Gradually, all the actors involved in the design process began to emphasise the benefits for the baby as well as the parents, referring to the positive responses of the parents and the ways in which Baby Watch played a role into integrating the baby in everyday life. The pictures of the babies were shown to siblings, grandparents, neighbours, friends, and people who generally do not come to a hospital. As a result, so it was concluded, the virtual babies became part of family life. One mother stated: "It is comforting to be a complete family for a while". "Besides the Royal Dutch Telephone account manager, there was also one of the nurses who emphasised the importance of Baby Watch for fathers: it would enable them to do something with the child and create a new way for men to deal with the situation. The image of the baby as profiting from Baby Watch thus played an important role in articulating the need and legitimising the introduction of Baby Watch in the hospital.

In addition to this legitimising function, images of the baby also figured in the various phases of the design process. The covers of two textual devices included pictures of the baby. The report published by Royal Dutch Telephone Research describing the experiences with the first prototype of Baby Watch includes an intriguing image of the baby: the baby is depicted as the user of Baby Watch. The cover shows a cartoon of a happy baby in diapers (the hair and the facial expressions suggest that it is a male baby) using a laptop, sitting next to a teddy bear. The manual of the viatv Phone system displays a strikingly different image of the baby. The cover shows two photographs: one of a baby lying on its stomach under a small blanket in the incubator, his/her face turned to the viewer. The second shows a man and a woman watching a $\mathrm{TV}$ showing a baby in an incubator. They are both smiling, and the woman is pointing to the baby on the screen. These images of the baby reflect the differences in the representation techniques used in various phases of the design process. The image of the playful baby exemplifies the distance between the world of $\mathrm{R} \& \mathrm{D}$ and the location of use that dominated the early phase of de- 
sign. As we have described above, visits by the designers to the site of use, i.e., the hospital and the home of the parents, only took place in a later stage of the design. The more realistic image of the baby on the cover of the Via tv Phone manual (realistic because they are photographs instead of cartoons, and portrayed the baby in the incubator rather than as a computer user) reflects a less remote relationship between the worlds of design and use.

The baby finally, figured into the design process when designers had to decide on the quality of the images of the baby on the screen. The specific circumstances of the incubator caused several technical problems. The incubator's white sheets had a particular influence on the camera's exposure. At this point in the design process, the baby was constructed as a patient, and the actual configuration of the situation was primarily oriented towards medical treatment. The camera had to be adjusted to this situation and so it was positioned in such a way that it would not interfere with other medical devices near the incubator. Moreover, the camera was designed to be very flexible and easy to handle by the nurses whenever the baby needed medical treatment. Again, the nurses acted as spokespersons for the babies.

\section{Nurses}

In contrast to the parents and babies, the nurses were more directly involved in the design process. As we described above, the senior nurse, and head of the neonatology ward and three other nurses participated in the project committee that attempted to control all stages of the design. Actually, the nurses performed three distinct roles in the design process. First, nurses acted as users. To them, the introduction of Baby Watch implied that they had to deal with a new and permanent element in their working environment. They had to take care of the babies in a highly technologised environment in which an additional device had been introduced, implying a change in their daily routines. As we have described above, the Baby Watch script delegated most of the responsibilities to the nurses who had to operate the system whenever the parents called, and they became responsible for a new form of "baby management" ("never lay a baby down in the wrong incubator!") as well as for the transmission of images. Second, the nurses played a crucial role in the dissemination of the new apparatus. It was their task to introduce Baby Watch to the parents and to give instructions and explanations on how to use the equipment. This was a new and complicated job because nurses had to negotiate with parents in a period in which the latter were overwhelmed by emotion and possibly grief and sorrow. To make things even more complicated, the nurses were rather ambivalent about Baby Watch because they thought 
the new technology might have a negative influence on the physical visits of the parents, as we described above. By involving the senior nurse in the design process, the designers were able to anticipate the ambivalence and possible resistance of nurses towards the new technology. Third, as has been described above, nurses functioned as spokespersons for the parents. In this case, the nurses acted as strong agents, as powerful actors. ${ }^{12}$

Because of these different roles, nurses became an "obligatory point of passage" for the designers, to use Bruno Latour's terminology, which explains why they were allowed to take part in negotiating the technological decisions (Latour 1987). Communication with the nurses was crucial for the designers: because the nurses provided them with information about the work floor and about the parents, and last but not least, they had to interest the parents in the new technology. The motivation of the nurses was essential to the success of the experiment because if the nurses did not want to work with Baby Watch, the technology would totally fail. This position enabled nurses to negotiate changes in the artifact to make the new equipment more convenient to use. In the second Baby Watch prototype, the inflexible camera was replaced by a flexible one as a reaction to complaints from nurses about the inconvenience of the fixed camera during the nursing of the babies.

\section{Hospital management}

Like the nurses, hospital management was also involved in negotiating technical decisions, although it did not participate in the special committee. This responsibility was delegated to the doctor who also had a seat on the committee. Although the designers considered parents as the users, the Royal Dutch Telephone project manager considered hospital as his primary customer. After all, it was the hospital that was paying for his company's services. The role of this fourth user became very crucial when the hospital manager first became alarmed by the unexpected consequences of the new technology. It was during an interview with the press that a journalist asked the manager of the hospital about possible abuses ever of Baby Watch. The recording of transmitted pictures could provide the parents with evidence if they ever wanted to start a legal process regarding the treatment of the baby. This unforeseen consequence produced a shock within the ranks of hospital management and elicited a new image of the end-user. Parents were suddenly portrayed as potential troublemakers because they could begin legal proceedings against the hospital regarding the treatment of their baby. As a result, the hospital management forbid the transmission of any images showing medical treatment. Consequently, new instructions for the nurses were formulated: during every 
medical treatment or nursing of a baby, they had to cover the camera with a lid, not only for the peace of mind of the parents, as nurses initially told us, but also in the general interest of the hospital. For the same reasons, and on the basis of another argument - the privacy of other patients and parents the transmission of sound was not an option in both the Baby Watch and the viATv Phone systems. In the latter system, the sound cable was physically removed. In the case of Magic $\mathrm{TV}$, used in the child oncology ward, the hospital management controlled the "virtual boundaries" of the hospital by using a separate room for video communication. Obviously, in the neonatology ward, this is impossible. The intervention of the hospital management thus resulted in a drastic change in the design of the viatv Phone system.

\section{Agency in influencing design}

Our study shows how multiple users were engaged in the design process, albeit in different ways. The nurses and hospital management had direct access to the design process; they could mobilise sufficient resources in order to influence the actual design and implementation of Baby Watch. The parents and babies, however, only took part in the design process indirectly. Their interests, wishes, and skills were assessed by spokespersons who acted on their behalf: the designers, the human-factor specialist, the account manager of Royal Dutch Telephone, and the nurses. They all had their own representations of the parents and the parents' wishes regarding their babies and/or Baby Watch. The parents themselves were interviewed only after the first prototype of the Baby Watch was introduced, which revealed that the representations of the users did not match the actual preferences and skills of the real users. Interestingly, the extent to which user groups were engaged in the design process is not congruent with the impact these groups had on the actual design. Although the nurses had direct access to the design process, their actual impact on the design was rather limited - only the design of a flexible camera was clearly influenced by the nurses' wishes. Only the babies had less impact. They shaped the design only indirectly through their parents' preference for the quality of the images on the screen, thus altering the design to include both moving and fixed images of the baby.

In comparison to the babies, the parents had a much larger impact on the design. Their resistance to using the initial Baby Watch prototype was a powerful influence on the characteristics of later artifacts: the system had to be open up to them on a permanent basis, and the "technological image" was masked by replacing the laptop with the more familiar TV. Clearly, a substantial group of parents did not accept the script of the initial Baby Watch proto- 
type. By rejecting the script, and thus becoming non-users, they motivated designers to change the design. The "talking back" of the users (Epstein 1996) resulted in the development of a new prototype. Although rejecting the script should thus be considered an important means to influence design, non-use does not guarantee that designers will adjust their designs to the wishes of end-users. The deterring influence of the technological image of a laptop, particularly with regard to ethnic groups, was judged by the designers to be an essential shortcoming of the system. However, the vi atv Phone system, in turn, also created non-users, although this group differed from the Baby Watch group. In the case of the viatv Phone, parents rejecting television on religious grounds were among the non-users. Strikingly, this type of exclusion was evaluated as being of "their own choice" and did not lead to design changes..$^{13}$

Finally, hospital management played an important role in the technological decision-making because they set the conditions of and the limitations to the transmission of signals and sounds. Their decision not to transmit sound overruled the interests of the parents. In negotiations on the design, the hospital manager's demand for limited access and transmission was more effective than the parents' wishes for open access. Although the designers considered the parents as the actual end-users, the technical decisions on what the new technology would eventually communicate to the parents were predominantly shaped by the demands and interests of hospital management. Its dual role as user and customer facilitated a situation in which Royal Dutch Telephone granted the hospital's preferences more authority than the parents' wishes.

\section{Conclusion}

Our script analysis of the various prototypes of this video-communication system illustrates how artifacts play an important role in delegating and distributing agency and control among the various users. The three artifacts used in the neonatology ward (that is two prototypes of Baby Watch and the viATv Phone) acted quite differently with respect to the way they distributed agency between different groups of users, i.e., parents and nurses, and between users and the artifact itself. Whereas the first prototype of Baby Watch delegated all responsibilities and control to the nurses and only limited agency to the parents, the second Baby Watch prototype delegated more agency to the parents. The viatv Phone system increased the agency of the parents further by adding the ability to control the image, although the par- 
ents still depended on the nurses to use the system. Most importantly, the parents were denied the ability to receive or send sound.

Our analysis thus suggests that the concept of distributed agency is an adequate tool to show that technological artifacts can contribute to an asymmetry in agency and control over technologies among heterogeneous groups of actors, human as well as nonhuman. The concept of distributed agency, however, does not perform very well if we want to understand the processes underlying the design of asymmetrical scripts. Whereas actor-network approaches emphasise the conceptual symmetry between human and nonhuman actors, this case study shows asymmetry: asymmetry between people and artifacts and, more importantly, asymmetry between various human actors. The problem is that the emphasis of actor-network theory upon the symmetry between humans and nonhumans tends to obscure asymmetries between human actors. ${ }^{14}$ Moreover, actor-network theory neglects an important conceptual difference between human and nonhuman agency. Although both people and artifacts can act, only humans can be held responsible for the technologies they produce. One cannot hold objects accountable for their actions - or perhaps one can, but this will not result in changes in design practices that create asymmetrical scripts. Most importantly, our case study shows that asymmetrical scripts are not the result of the agency of nonhuman actors but originate from human decisions. The agency of parents to operate and co-produce the video communication products remained relatively limited, not because of technical constraints but because of negotiations and power relations between the different actors involved in the design process. Hospital management overruled the possible interactivity of the artifact by adding a camera lid and by disconnecting the camera's sound cable. This decision overruled the interests of the parents. In accordance with the existing literature on the influence of the various user groups on design processes, we found that the end-users were assigned less agency than the other groups involved in the design process (Berg et al. 1999; Lehoux et al. 1999; Clarke and Montini 1993).

We therefore conclude that, in contrast to what Latour suggests, actions do have a point of origin (Latour $1996^{\mathrm{a}}, 237-8$ ). In our case study, hospital management and the designers could mobilise resources to boost their own interests in the design and implementation process. Their interventions restricted the possibilities of the technology and the possibilities/agency of the parents, but only the latter were in a position to react directly to interventions. They did not like the technology of the first prototype and were reluctant to use it as a means of communication with their babies, which forced the designers to adapt the system. This kind of more or less deliberate action is human-based. 
At this point in the analysis, the usefulness and analytical strength of the concept of symmetry between human and nonhuman actors comes to an end. Our study shows that "symmetry" is primarily a very useful analytical instrument but also that, in many cases, human actors make the difference. To be able to answer questions concerning the politics of technology, we need to acknowledge that, ultimately, human and nonhuman agencies do not coincide and should be considered as different entities.

\section{Notes}

1 Latour $\left(1996^{\mathrm{a}}\right)$ defines this in his article "On Interobjectivity" as "to act to mediate another's action". Later, in his reply to critics, he again distances himself from the concept of mediation.

2 In 1998, we organised a workshop for the Royal Dutch Telephone Company, the erstwhile market leader in communication technology in the Netherlands. The workshop aimed to discuss user-oriented design processes and the actual design practices of Royal Dutch Telephone. We decided to take one case and reconstruct the design and implementation process, and the way users were configured and engaged in these processes. Baby Watch seemed to be a useful case study because the technology was developed for multiple users, and different user groups were involved in the design process. Before the workshop, we interviewed the relevant staff members: the account manager, the human-factor specialist, and the software designer. In addition to the interviews, we used several reports written by Royal Dutch Telephone during the design of this video communication system.

3 Interview Royal Dutch Telephone account manager, February 5, 1998.

4 See Akrich (1995) for this distinction between implicit, informal techniques and explicit, formal techniques to assess user needs. Explicit representation techniques, such as marketing surveys, consumer tests and feedback from users through after-sales services, are legitimised by formal, scientific bases, whereas informal techniques lack this basis. Examples of the latter are reliance on personal experience, on the consultancy of experts, and on user images incorporated into earlier products.

5 Interview with the head of neonatology, January $27,1998$.

6 Interview with a designer from Royal Dutch Telephone, February 5, 1998.

7 After some months, the interface became a little more complex, since the system was used in locations where the pre-installed telephone number did not work, e.g., in Germany and internally in the hospital. The parents first had to work through some menus before they could start the system.

8 Interview with the head of neonatology, January 27, 1998. 
9 Interview with the account manager of Royal Dutch Telephone, February 5, 1998.

10 Interview with a designer from Royal Dutch Telephone, February 5, 1998.

11 This representation of the family and the baby was also included in some of the newspapers which reacted to the press report launched by the Radboud Hospital after several weeks of using Baby Watch (Anonymous 1996; Knol 1996).

12 Until recently, sociological research on hospitals displayed a rather surprising absence of nurse-related information, although their work is part of the core business of medical organisations. Research from a gender perspective revealed the importance of the nurse as a powerful actor within hospitals (see, for instance, Davies 1995).

13 Interview with the head of neonatology, January 27, 1998.

14 A similar critique has been articulated by other scholars, most notably Adele Clarke. Clarke has introduced the concept of "implicated actor" to avoid silencing invisible actors and to include power relations in the analysis of user-expert relationships. This concept includes two categories of actors: "those not physically present but who are discursively constructed and targeted by others," and "those who are physically present but who are generally silenced/ignored/made invisible by those in power". (Clarke 2004; see also Clarke and Montini 1993; Clarke 1998). Our case study exemplifies the second category. 